\title{
ANALYSIS OF NETWORKS WITH TIME-DEPENDENT TRANSITION PROBABILITIES AND MESSAGES BYPASS BETWEEN THE QUEUING SYSTEMS
}

\author{
Mikhail Matalytski ${ }^{1}$, Victor Naumenko ${ }^{2}$ \\ ${ }^{I}$ Institute of Mathematics, Czestochowa University of Technology, Czestochowa, Poland \\ ${ }^{2}$ Grodno State University, Grodno, Belarus \\ m.matalytski@gmail.com,victornn86@gmail.com
}

\begin{abstract}
The object of investigation is an open exponential network with a messages bypass of systems in transient behavior. The purpose of the research is to find stationary probabilities of states and the average characteristics of the network when the transition probabilities between the messages and bypass systems of the network, parameters of the incoming flow of messages and services are time-dependent. To find the state probabilities and the characteristics of a network is used the apparatus for the multivariate generating functions. The examples are calculated on a computer.
\end{abstract}

\section{General information}

The results of research of the above networks in stationary behavior are given in [1-3]. In [4] investigated the network in a transition behavior, but when the probability messages bypass between systems, networks do not depend on network status and time. To find the state probabilities of the network in a transition mode, we used the method of multidimensional generating functions, which was previously used to find the state probabilities of the other networks $[5,6]$. In this paper we consider the case when the transition probabilities and messages bypass between the network systems depend on time.

\section{Formulation of the problem}

Consider an open exponential QN of arbitrary structure consisting of $n$ QS, enumerated by numbers from 1 to $n$. Messages have a chance to join the queue, and with an additional probability to move immediately in accordance with the transition probability matrix of the other QS, or leave the network. The probability of joining the QS depends on the state of the QS and the number of QS with which the messages are sent to this QS. It is assumed that the incoming flow of applications to the network is simple. The results of studies of such networks in the steady state are given in [1-4]. This paper describes a method of finding the timedependent state probabilities of the network of such a network in the transient state. 
Let $m_{i}$ - number of identical service lines in the QS $S_{i}, I_{i}$ - a vector of dimension $n$, consisting of zeros except the $i$-th component, which is equal to $1, i=\overline{1, n}$; $p_{i j}$ - the transition probability of the message after service in the system $S_{i}$ into the system $S_{j}, i, j=\overline{0, n}$, we assume the system $S_{0}$ is the external environment. Let us consider the case when the parameters of the incoming flow of messages and services depend on time, i.e. the time interval $[t, t+\Delta t)$ in the network receives an message with a probability $\lambda(t) \Delta t+o(\Delta t)$, and if at the time $t$ of service on the line $i$-th QS is located an message, at the range $[t, t+\Delta t)$ of its services will end with a probability $\mu_{i}(t) \Delta t+o(\Delta t), i=\overline{1, n}$. The message is sent to the $i$-th QS with probability $p_{0 i}, \sum_{i=1}^{n} p_{0 i}=1$. The message sent to this QS from the external environment at moment time $t$, with a probability $f^{(i)}(k, t)$, when the network is in a state $(k, t)$, joins the queue, and the probability $1-f^{(i)}(k, t)$ is not attached to the queue, regardless of handled (i.e., it's time of service with a probability of 1 is equal to zero). If the message has been served in the $i$-th QS, it is likely to be sent immediately to the $j$-th QS with probability $p_{i j}$, and leaves the QN with the probability $p_{i 0}, \sum_{j=0}^{n} p_{i j}=1, i=1, \ldots, n$.

Let $k(t)=(k, t)=\left(k_{1}, k_{2}, \ldots, k_{n}, t\right)$ - the state vector of the network, where $k_{i}$ - the number of messages at the moment $t$ in the system $S_{i}, i=\overline{1, n} ; \varphi_{i}(k, t)$ - the conditional probability that the message is delivered to the $i$-th QS at time $t$, when the network is in a state $(k, t)$, will not be serviced by any of the QS; $\psi_{i j}(k, t)$ - the conditional probability that the message is delivered to the $i$-th QS outside at time $t$, when the network is in state $(k, t)$, the first time, a service in $j$-th QS; $\alpha_{i}(k, t)$ - the conditional probability that the message, served in the $i$-th queuing system at time $t$, when the network is in a state $(k, t)$, will no longer be served in any of QS; $\beta_{i j}(k, t)$ - the conditional probability that the message, served in the $i$-th queuing system at time $t$, when the network is in state $(k, t)$ for the first time then receive services in the $j$-th QS, $i, j=\overline{1, n}$.

According to the formula of total probability, we obtain:

$$
\begin{gathered}
\varphi_{i}(k, t)=\left(1-f^{(i)}(k, t)\right)\left(p_{i 0}+\sum_{j=1}^{n} p_{i j} \varphi_{j}(k, t)\right), i=\overline{1, n}, \\
\psi_{i j}(k, t)=f^{(i)}(k, t) \delta_{i j}+\left(1-f^{(i)}(k, t)\right) \sum_{l=1}^{n} p_{i l} \psi_{l j}(k, t), i, j=\overline{1, n},
\end{gathered}
$$




$$
\begin{gathered}
\alpha_{i}(k, t)=p_{i 0}+\sum_{j=1}^{n} p_{i j} \varphi_{j}\left(k-I_{i}, t\right), \quad i=\overline{1, n}, \\
\beta_{i j}(k, t)=\sum_{l=1}^{n} p_{i l} \psi_{l j}\left(k-I_{i}, t\right), i, j=\overline{1, n},
\end{gathered}
$$

where $\delta_{i j}$ - the Kronecker delta. We have the equalities

$$
\alpha_{i}(k, t)=1-\sum_{j=1}^{n} \beta_{i j}(k, t), \varphi_{i}(k, t)+\sum_{j=1}^{n} \psi_{i j}(k, t)=1, i, j=\overline{1, n} .
$$

From (1) and (3) we find

$$
\psi_{i j}(k, t)=f^{(i)}(k, t) \delta_{i j}+\left(1-f^{(i)}(k, t)\right) \beta_{i j}\left(k-I_{i}, t\right), i, j=\overline{1, n} .
$$

The probabilities of states of the network under consideration satisfy the difference-differential equations (DDE) is proved in [4]:

$$
\begin{aligned}
& \frac{d P(k, t)}{d t}=-\sum_{i=1}^{n} {\left[\lambda(t) p_{0 i}\left(1-\varphi_{i}(k, t)\right)+\mu_{i}(t)\left(1-\beta_{i i}(k, t)\right) \min \left(m_{i}, k_{i}\right)\right] P(k, t)+} \\
&+\lambda(t) \sum_{i=1}^{n} \sum_{j=1}^{n} p_{0 i} \psi_{i j}\left(k-I_{j}, t\right) u\left(k_{i}\right) P\left(k-I_{j}, t\right)+ \\
&+\sum_{i=1}^{n} \mu_{i}(t) \min \left(m_{i}, k_{i}+1\right) \alpha_{i}\left(k+I_{i}, t\right) P\left(k+I_{i}, t\right)+ \\
&+\sum_{\substack{i, j=1 \\
i \neq j}}^{n} \mu_{i}(t) \min \left(m_{i}, k_{i}+1\right) \beta_{i j}\left(k+I_{i}-I_{j}, t\right) u\left(k_{j}\right) P\left(k+I_{i}-I_{j}, t\right),
\end{aligned}
$$

where $u(x)=\left\{\begin{array}{l}1, x>0 \\ 0, x \leq 0\end{array}\right.$ is the Heaviside function.

\section{Finding the state probabilities}

Let $m_{i}=1, i=\overline{1, n}$, and suppose that all network system operating in high load mode, i.e. $k_{i}(t)>0 \quad \forall t>0, i=\overline{1, n}$, then the system (5) takes the form

$$
\begin{gathered}
\frac{d P(k, t)}{d t}=-\sum_{i=1}^{n}\left[\lambda(t) p_{0 i}\left(1-\varphi_{i}(k, t)\right)+\mu_{i}(t)\left(1-\beta_{i i}(k, t)\right)\right] P(k, t)+ \\
+\lambda(t) \sum_{i=1}^{n} \sum_{j=1}^{n} p_{0 i} \psi_{i j}\left(k-I_{j}, t\right) P\left(k-I_{j}, t\right)+\sum_{i=1}^{n} \mu_{i}(t) \alpha_{i}\left(k+I_{i}, t\right) P\left(k+I_{i}, t\right)+ \\
+\sum_{\substack{i, j=1 \\
i \neq j}}^{n} \mu_{i}(t) \beta_{i j}\left(k+I_{i}-I_{j}, t\right) P\left(k+I_{i}-I_{j}, t\right) .
\end{gathered}
$$


Note, that the number of equations in (6) is countable, when the network is open, and of course, when it is closed.

We denote by $\Psi_{n}(z, t)$, where $z=\left(z_{1}, z_{2}, \ldots, z_{n}\right), n$-dimensional generating function:

$$
\Psi_{n}(z, t)=\sum_{k_{1}=0}^{\infty} \sum_{k_{2}=0}^{\infty} \ldots \sum_{k_{n}=0}^{\infty} P\left(k_{1}, k_{2}, . ., k_{n}, t\right) z_{1}^{k_{1}} z_{2}^{k_{2}} \cdot \ldots \cdot z_{n}^{k_{n}}=\sum_{k_{1}=1}^{\infty} \sum_{k_{2}=1}^{\infty} \ldots \sum_{k_{n}=1}^{\infty} P(k, t) \prod_{i=1}^{n} z_{i}^{k_{i}}
$$

the summation is taken over each $k_{i}$, from 1 to $\infty, i=\overline{1, n}$, because the network is operating in a high load mode.

Consider the case where the conditional probabilities $\varphi_{i}(k, t), \psi_{i j}(k, t), \alpha_{i}(k, t)$, $\beta_{i j}(k, t)$ do not depend on the state of the network. The expression for the generating function (7) can be rewritten as

$$
\begin{gathered}
\Psi_{n}(z, t)=C_{n}(z) \exp \left\{-\int \sum_{i=1}^{n}\left[\lambda(t) p_{0 i}\left(1-\varphi_{i}(t)\right)+\mu_{i}(t)\left(1-\beta_{i i}(t)\right)+\right.\right. \\
\left.\left.+\lambda(t) p_{0 i} z_{i} \sum_{\mathrm{j}=1}^{n} \psi_{i j}(t)+\mu_{i}(t) \frac{\alpha_{i}(t)}{z_{i}}+\mu_{i}(t) \sum_{j=1}^{n} \beta_{i j}(t) \frac{z_{j}}{z_{i}}\right] d t\right\},
\end{gathered}
$$

where the function $C_{n}(z)$ was defined in the proof of Lemma 2 in paper [4], from the conditions, that at the initial time the network is able to $\left(x_{1}, x_{2}, \ldots, x_{n}, 0\right), x_{i}>0$, $i=\overline{1, n}, P\left(x_{1}, x_{2}, \ldots, x_{n}, 0\right)=1, P\left(k_{1}, k_{2}, \ldots, k_{n}, 0\right)=0, \forall x_{i} \neq k_{i}, i=\overline{1, n}$ :

$$
\begin{gathered}
C_{n}(z)=\exp \left\{\Lambda(0) \sum_{i=1}^{n} p_{0 i}-\sum_{i=1}^{n} p_{0 i} \Phi_{i}(0)+\sum_{i=1}^{n} M_{i}(0)-\sum_{i=1}^{n} \mathrm{~B}_{i i}(0)+\right. \\
\left.+\sum_{i=1}^{n} p_{0 i} z_{i} \sum_{\mathrm{j}=1}^{n} \mathrm{Y}_{i j}(0)+\sum_{i=1}^{n} \frac{1}{z_{i}} \mathrm{~A}_{i}(0)+\sum_{i=1}^{n} \sum_{j=1}^{n} \frac{z_{j}}{z_{i}} \mathrm{~B}_{i j}(0)\right\} \prod_{i=1}^{n} z_{l}^{x_{l}} .
\end{gathered}
$$

Let us introduce the following notations:

$$
\begin{gathered}
\Lambda(t)=\int \lambda(t) d t, M_{i}(t)=\int \mu_{i}(t) d t, \Phi_{i}(t)=\int \lambda(t) \varphi_{i}(t) d t, \mathrm{Y}_{i j}(t)=\int \lambda(t) \psi_{i j}(t) d t, \\
\mathrm{~A}_{i}(t)=\int \mu_{i}(t) \alpha_{i}(t) d t, \mathrm{~B}_{i j}(t)=\int \mu_{i}(t) \beta_{i j}(t) d t .
\end{gathered}
$$

Using the notation (10), expression (8) can be rewritten as

$$
\begin{aligned}
\Psi_{n}(z, t)= & C_{n}(z) \exp \left\{-\left(\Lambda(t) \sum_{i=1}^{n} p_{0 i}-\sum_{i=1}^{n} p_{0 i} \Phi_{i}(t)+\sum_{i=1}^{n} M_{i}(t)-\sum_{i=1}^{n} \mathrm{~B}_{i i}(t)+\right.\right. \\
& \left.\left.+\sum_{i=1}^{n} p_{0 i} z_{i} \sum_{\mathrm{j}=1}^{n} \mathrm{Y}_{i j}(t)+\sum_{i=1}^{n} \frac{1}{z_{i}} \mathrm{~A}_{i}(t)+\sum_{i=1}^{n} \sum_{j=1}^{n} \frac{z_{j}}{z_{i}} \mathrm{~B}_{i j}(t)\right)\right\} .
\end{aligned}
$$


Then from (9) and (11) that

$$
\begin{gathered}
\Psi_{n}(z, t)=\exp \left\{-\left((\Lambda(t)-\Lambda(0)) \sum_{i=1}^{n} p_{0 i}-\sum_{i=1}^{n} p_{0 i}\left(\Phi_{i}(t)-\Phi_{i}(0)\right)+\sum_{i=1}^{n}\left(M_{i}(t)-M_{i}(0)\right)-\right.\right. \\
-\sum_{i=1}^{n}\left(\mathrm{~B}_{i i}(t)-\mathrm{B}_{i i}(0)\right)+\sum_{i=1}^{n} p_{0 i} z_{i} \sum_{\mathrm{j}=1}^{n}\left(\mathrm{Y}_{i j}(t)-\mathrm{Y}_{i j}(0)\right)+\sum_{i=1}^{n} \frac{1}{z_{i}}\left(\mathrm{~A}_{i}(t)-\mathrm{A}_{i}(0)\right)+ \\
\left.\left.+\sum_{i=1}^{n} \sum_{j=1}^{n} \frac{z_{j}}{z_{i}}\left(\mathrm{~B}_{i j}(t)-\mathrm{B}_{i j}(0)\right)\right)\right\} \prod_{i=1}^{n} z_{l}^{x_{l}} .
\end{gathered}
$$

This expression can be rewritten as

$$
\begin{gathered}
\Psi_{n}(z, t)=a_{0}(t) \exp \left\{\sum_{i=1}^{n} p_{0 i} z_{i} \sum_{\mathrm{j}=1}^{n}\left(\mathrm{Y}_{i j}(t)-\mathrm{Y}_{i j}(0)\right)\right\} \times \\
\times \exp \left\{\sum_{i=1}^{n} \frac{1}{z_{i}}\left(\mathrm{~A}_{i}(t)-\mathrm{A}_{i}(0)\right)\right\} \exp \left\{\sum_{i=1}^{n} \sum_{j=1}^{n} \frac{z_{j}}{z_{i}}\left(\mathrm{~B}_{i j}(t)-\mathrm{B}_{i j}(0)\right)\right\} \prod_{i=1}^{n} z_{l}^{x_{l}} \\
a_{0}(t)=\exp \left\{-\sum_{i=1}^{n}\left[\left((\Lambda(t)-\Lambda(0))-\left(\Phi_{i}(t)-\Phi_{i}(0)\right)\right) p_{0 i}+\right.\right. \\
\left.\left.+\left(M_{i}(t)-M_{i}(0)\right)-\left(\mathrm{B}_{i i}(t)-\mathrm{B}_{i i}(0)\right)\right]\right\} .
\end{gathered}
$$

Transform (12) to a form suitable for finding the state probabilities of the network, expanding its member exponential in a Maclaurin series. From (7) and (12) that

$$
\Psi_{n}(z, t)=a_{0}(t) a_{1}(z, t) a_{2}(z, t) a_{3}(z, t) \prod_{l=1}^{n} z_{l}^{x_{l}},
$$

where

$$
\begin{aligned}
& a_{1}(z, t)=\exp \left\{\sum_{i=1}^{n} p_{0 i} z_{i} \sum_{\mathrm{j}=1}^{n}\left(\mathrm{Y}_{i j}(t)-\mathrm{Y}_{i j}(0)\right)\right\}=\prod_{i=1}^{n} \prod_{j=1}^{n} \exp \left\{p_{0 i} z_{i}\left(\mathrm{Y}_{i j}(t)-\mathrm{Y}_{i j}(0)\right)\right\}= \\
& =\prod_{i=1}^{n} \prod_{j=1}^{n} \sum_{l_{i}=0}^{\infty} \frac{\left[p_{0 i} z_{i}\left(\mathrm{Y}_{i j}(t)-\mathrm{Y}_{i j}(0)\right)\right]^{i_{i}}}{l_{i} !}=\sum_{l_{1}=0}^{\infty} \ldots \sum_{l_{n}=0}^{\infty} \prod_{i=1}^{n} \prod_{j=1}^{n} \frac{\left[p_{0 i} z_{i}\left(\mathrm{Y}_{i j}(t)-\mathrm{Y}_{i j}(0)\right)\right]^{k_{i}}}{l_{i} !}= \\
& =\sum_{l_{1}=0}^{\infty} \cdots \sum_{l_{n}=0}^{\infty} \frac{p_{01}^{l_{1}} \cdots p_{0 n}^{l_{n}} z_{1}^{l_{1}} \cdots z_{n}^{l_{n}}}{l_{1} ! l_{2} ! \cdots l_{n} !} \prod_{i=1}^{n} \prod_{j=1}^{n}\left[\left(\mathrm{Y}_{i j}(t)-\mathrm{Y}_{i j}(0)\right)\right]^{i}, \\
& a_{2}(z, t)=\exp \left\{\sum_{i=1}^{n} \frac{1}{z_{i}}\left(\mathrm{~A}_{i}(t)-\mathrm{A}_{i}(0)\right)\right\}=\prod_{i=1}^{n} \exp \left\{\frac{1}{z_{i}}\left(\mathrm{~A}_{i}(t)-\mathrm{A}_{i}(0)\right)\right\}= \\
& =\prod_{i=1}^{n} \sum_{q_{i}=0}^{\infty} \frac{\left[\left(\mathrm{A}_{i}(t)-\mathrm{A}_{i}(0)\right) z_{i}^{-1}\right]^{q_{i}}}{q_{i} !}=\sum_{q_{1}=0}^{\infty} \ldots \sum_{q_{n}=0}^{\infty} \prod_{i=1}^{n} \frac{\left[\left(\mathrm{A}_{i}(t)-\mathrm{A}_{i}(0)\right) z_{i}^{-1}\right]^{q_{i}}}{q_{i} !}= \\
& \sum_{q_{1}=0}^{\infty} \ldots \sum_{q_{n}=0}^{\infty} \frac{\prod_{i=1}^{n}\left[\mathrm{~A}_{i}(t)-\mathrm{A}_{i}(0)\right]^{q_{i}}}{q_{1} ! \cdot \ldots \cdot q_{n} !} z_{1}^{-q_{1}} \cdots z_{n}^{-q_{n}}
\end{aligned}
$$




$$
\begin{gathered}
a_{3}(z, t)=\exp \left\{\sum_{i=1}^{n} \sum_{j=1}^{n} \frac{z_{j}}{z_{i}}\left(\mathrm{~B}_{i j}(t)-\mathrm{B}_{i j}(0)\right)\right\}= \\
=\prod_{i=1}^{n} \prod_{j=1}^{n} \exp \left\{\frac{z_{j}}{z_{i}}\left(\mathrm{~B}_{i j}(t)-\mathrm{B}_{i j}(0)\right)\right\}=\prod_{i=1}^{n} \prod_{j=1}^{n} \sum_{r_{i}=0}^{\infty} \frac{\left[\left(\mathrm{B}_{i j}(t)-\mathrm{B}_{i j}(0)\right) z_{j} z_{i}^{-1}\right]^{r_{i}}}{r_{i} !}= \\
=\sum_{r_{1}=0}^{\infty} \cdot \sum_{r_{n}=0}^{\infty} \prod_{i=1}^{n} \prod_{j=1}^{n} \frac{\left[\left(\mathrm{B}_{i j}(t)-\mathrm{B}_{i j}(0)\right) z_{j} z_{i}^{-1}\right]^{r_{i}}}{r_{i} !}=\prod_{i=1}^{n} \prod_{j=1}^{n} \exp \left\{\frac{\left.z_{j}\left(\mathrm{~B}_{i j}(t)-\mathrm{B}_{i j}(0)\right)\right\}=}{z_{i}}=\right. \\
=\prod_{i=1}^{n} \prod_{j=1}^{n} \sum_{r_{i}=0}^{\infty} \frac{\left[\left(\mathrm{B}_{i j}(t)-\mathrm{B}_{i j}(0)\right) z_{j} z_{i}^{-1}\right]_{j}^{r_{i}}}{r_{i} !}=\sum_{r_{1}=0}^{\infty} \cdot \sum_{r_{n}=0}^{\infty} \prod_{i=1}^{n} \prod_{j=1}^{n}\left[\left(\mathrm{~B}_{i j}(t)-\mathrm{B}_{i j}(0)\right) z_{j} z_{i}^{-1}\right]_{i}^{r_{i}} \\
=\sum_{r_{1}=0}^{\infty} \ldots \sum_{r_{n}=0}^{\infty} \frac{\prod_{i=1}^{n}\left(\prod_{j=1}^{n}\left(\mathrm{~B}_{i j}(t)-\mathrm{B}_{i j}(0)\right)\right)^{r_{i}}}{r_{1} ! \cdots r_{n} !} z_{1}^{r_{1}+r_{2}+\ldots+r_{n}} z_{2}^{r_{1}+r_{2}+\ldots+r_{n}} \ldots z_{n}^{r_{1}+r_{2}+\ldots+r_{n}} z_{1}^{-r_{1}} z_{2}^{-r_{2}} \ldots z_{n}^{-r_{n}}= \\
=\sum_{r_{1}=0}^{\infty} \ldots \sum_{r_{n}=0}^{\infty} \frac{\prod_{i=1}^{n}\left(\prod_{j=1}^{n}\left(\mathrm{~B}_{i j}(t)-\mathrm{B}_{i j}(0)\right)\right)^{r_{i}}}{r_{1} ! \cdots r_{n} !} z_{1}^{R-r_{1}} \ldots z_{n}^{R-r_{n}}
\end{gathered}
$$

Multiplying $a_{0}(t), a_{1}(z, t), a_{2}(z, t), a_{3}(z, t)$ and $\prod_{l=1}^{n} z_{l}^{x_{l}}$ obtain the expression for the generating function (7) has the form:

$$
\begin{gathered}
\Psi_{n}(z, t)=a_{0}(t) \sum_{l_{1}=0}^{\infty} \ldots \sum_{l_{n}=0}^{\infty} \sum_{q_{1}=0}^{\infty} \ldots \sum_{q_{n}=0 r_{1}=0}^{\infty} \ldots \sum_{r_{n}=0}^{\infty} \prod_{i=1}^{n}\left[\frac{p_{0 i}^{l_{i}}}{l_{i} ! q_{i} ! r_{i} !} \times\right. \\
\left.\times\left(\prod_{j=1}^{n}\left(\mathrm{Y}_{i j}(t)-\mathrm{Y}_{i j}(0)\right)\right)^{l_{i}}\left(\mathrm{~A}_{i}(t)-\mathrm{A}_{i}(0)\right)^{q_{i}} \times\left(\prod_{j=1}^{n}\left(\mathrm{~B}_{i j}(t)-\mathrm{B}_{i j}(0)\right)\right)^{r_{i}} z_{i}^{x_{i}+l_{i}-q_{i}-r_{i}+R}\right],
\end{gathered}
$$

where $R=\sum_{i=1}^{n} r_{i}$

\section{Finding the average number of messages in the network systems}

It is known that the expectation of the $c$-th component of a multidimensional random variable can be found by differentiating the generating function (13) and putting on $z_{i}=1, i=\overline{1, n}$. Therefore, the average number of messages in system $S_{c}$ will use the relation: 


$$
\begin{gathered}
\frac{\partial \Psi_{n}(z, t)}{\partial z_{c}}=a_{0}(t) \sum_{l_{1}=0}^{\infty} \ldots \sum_{l_{n}=0}^{\infty} \sum_{q_{1}=0}^{\infty} \ldots \sum_{q_{n}=0 r_{1}=0}^{\infty} \ldots \sum_{r_{n}=0}^{\infty}\left(x_{c}+l_{c}-q_{c}-r_{c}+R\right) \times \\
\times \prod_{i=1}^{n}\left[\frac{p_{0 i}^{l_{i}}}{l_{i} ! q_{i} ! r_{i} !}\left(\prod_{j=1}^{n}\left(\mathrm{Y}_{i j}(t)-\mathrm{Y}_{i j}(0)\right)\right)^{l_{i}}\left(\mathrm{~A}_{i}(t)-\mathrm{A}_{i}(0)\right)^{q_{i}}\left(\prod_{j=1}^{n}\left(\mathrm{~B}_{i j}(t)-\mathrm{B}_{i j}(0)\right)\right)^{r_{i}}\right] \times \\
\times z_{c}^{x_{c}+l_{c}-q_{c}-r_{c}+R-1} \prod_{\substack{i=1, i \neq c}}^{n} z_{i}^{x_{i}+l_{i}-q_{i}-r_{i}+R} .
\end{gathered}
$$

It follows that the average number of applications in the system $S_{c}$ is determined by the formula

$$
\begin{aligned}
& N_{c}(t)=\left.\frac{\partial \Psi_{n}(z, t)}{\partial z_{c}}\right|_{z=(1,1, \ldots, 1)}=a_{0}(t) \sum_{l_{1}=0}^{\infty} \ldots \sum_{l_{n}=0 q_{1}=0}^{\infty} \sum_{q_{n}=0 r_{1}=0}^{\infty} \ldots \sum_{r_{n}=0}^{\infty}\left(x_{c}+l_{c}-q_{c}-r_{c}+R\right) \times \\
& \left.\times \prod_{i=1}^{n}\left[\frac{p_{0 i}^{l_{i}}}{l_{i} ! q_{i} ! r_{i} !}\left(\prod_{j=1}^{n}\left(\mathrm{Y}_{i j}(t)-\mathrm{Y}_{i j}(0)\right)\right)\right)^{l_{i}}\left(\mathrm{~A}_{i}(t)-\mathrm{A}_{i}(0)\right)^{q_{i}}\left(\prod_{j=1}^{n}\left(\mathrm{~B}_{i j}(t)-\mathrm{B}_{i j}(0)\right)\right)^{r_{i}}\right] .(14)
\end{aligned}
$$

We make in (14) change of variables $k_{c}=x_{c}+l_{c}-q_{c}-r_{c}+R$, then $l_{c}=k_{c}-x_{c}+q_{c}+r_{c}-R$ and

$$
\begin{gathered}
N_{c}(t)=a_{0}(t) \sum_{q_{1}=0}^{\infty} \ldots \sum_{q_{n}=0 r_{1}=0}^{\infty} \ldots \sum_{r_{n}=0}^{\infty} \sum_{k_{1}=x_{1}-q_{1}-r_{1}+R}^{\infty} \ldots \sum_{k_{n}=x_{n}-q_{n}-r_{n}+R}^{\infty} k_{n_{i}-x_{i}} \times \\
\times \prod_{i=1}^{n}\left[\frac{p_{0 i}^{l_{i}}}{\left(k_{i}-x_{i}+q_{i}+r_{i}-R\right) ! q_{i} ! r_{i} !}\left(\prod_{j=1}^{n}\left(\mathrm{Y}_{i j}(t)-\mathrm{Y}_{i j}(0)\right)\right)^{k_{i}-q_{i}+q_{i}+r_{i}-R} \times\right. \\
\left.\times\left(\mathrm{A}_{i}(t)-\mathrm{A}_{i}(0)\right)^{q_{i}}\left(\prod_{j=1}^{n}\left(\mathrm{~B}_{i j}(t)-\mathrm{B}_{i j}(0)\right)\right)^{r_{i}}\right] .
\end{gathered}
$$

Because the network operating system under high load mode $k_{i}=x_{i}-q_{i}-r_{i}+R \geq 1$ and, therefore, $q_{i} \leq x_{i}-r_{i}+R-1$, thus

$$
\begin{gathered}
N_{c}(t)=a_{0}(t) \sum_{k_{1}=1}^{\infty} \ldots \sum_{k_{n}=1}^{\infty} k_{c} \sum_{r_{1}=0}^{\infty} \ldots \sum_{r_{n}=0}^{\infty} \sum_{q_{1}=0}^{x_{1}-r_{i}+R-1} \cdots \sum_{q_{n}=0}^{x_{n}-r_{n}+R-1} \times \\
\times \prod_{i=1}^{n}\left[\frac{p_{0 i}^{l_{i}}\left[\frac{x_{i}}{\left(k_{i}-x_{i}+q_{i}-R\right) ! q_{i} ! r_{i} !}\left(\prod_{j=1}^{n}\left(\mathrm{Y}_{i j}(t)-\mathrm{Y}_{i j}(0)\right)\right)^{k_{i}-x_{i}+q_{i}+r_{i}-R} \times\right.}{\left.\times\left(\mathrm{A}_{i}(t)-\mathrm{A}_{i}(0)\right)^{q_{i}}\left(\prod_{j=1}^{n}\left(\mathrm{~B}_{i j}(t)-\mathrm{B}_{i j}(0)\right)\right)^{r_{i}}\right]} .\right.
\end{gathered}
$$




\section{Example 1}

Let the intensity $\lambda(t)=\lambda t, \quad \mu_{i}(t)=\mu_{i}\left[\cos \left(\omega_{i} t\right)+1\right], i=\overline{1, n}$. In this case $\Lambda(t)=\frac{\lambda}{2} t^{2}, \Lambda(0)=0, M_{i}(t)=\mu_{i}\left[\frac{\sin \left(\omega_{i} t\right)}{\omega_{i}}+t\right], M_{i}(0)=0, i=\overline{1, n}$. Suppose, that the probability of adherence message to the queue at time $t$ is given by $f^{(i)}(t)=1-e^{-i t}$. From (10) follows that

$$
\begin{gathered}
\Phi_{i}(t)=\int \lambda(t) \varphi_{i}(t) d t=\int \varphi_{i}(t) d \Lambda(t)=\varphi_{i}(t) \Lambda(t)-\int \varphi_{i}^{\prime}(t) \Lambda(t) d t, \\
\mathrm{Y}_{i j}(t)=\int \lambda(t) \psi_{i j}(t) d t=\int \psi_{i j}(t) d \Lambda(t)=\psi_{i j}(t) \Lambda(t)-\int \psi_{i j}^{\prime}(t) \Lambda(t) d t, \\
\mathrm{~A}_{i}(t)=\int \mu_{i}(t) \alpha_{i}(t) d t=\int \alpha_{i}(t) d M_{i}(t)=\alpha_{i}(t) M_{i}(t)-\int \alpha_{i}^{\prime}(t) M_{i}(t) d t, \\
\mathrm{~B}_{i j}(t)=\int \mu_{i}(t) \beta_{i j}(t) d t=\int \beta_{i j}(t) d M_{i}(t)=\beta_{i j}(t) M_{i}(t)-\int \beta_{i j}^{\prime}(t) M_{i}(t) d t,
\end{gathered}
$$

and we get that

$$
\begin{gathered}
a_{0}(t)=\exp \left\{-\sum_{i=1}^{n}\left[\frac{\lambda}{2}\left(t^{2}-\varphi_{i}(t) t^{2}+\int \varphi_{i}^{\prime}(t) t^{2} d t\right) p_{0 i}+\right.\right. \\
\left.\left.+\mu_{i}\left(\frac{\sin \left(\omega_{i} t\right)}{\omega_{i}}+t-\beta_{i i}(t)\left(\frac{\sin \left(\omega_{i} t\right)}{\omega_{i}}+t\right)+\int \beta_{i i}^{\prime}(t)\left(\frac{\sin \left(\omega_{i} t\right)}{\omega_{i}}+t\right) d t\right)\right\}\right]= \\
=\exp \left\{-\sum_{i=1}^{n}\left[\frac{\lambda}{2}\left(t^{2}-\int \varphi_{i}(t) t d t\right) p_{0 i}+\mu_{i}\left(\frac{\sin \left(\omega_{i} t\right)}{\omega_{i}}+t-\int \beta_{i i}(t)\left(\cos \left(\omega_{i} t\right)+1\right) d t\right)\right]\right\},
\end{gathered}
$$

$\Phi_{i}(0)=0, \quad \mathrm{Y}_{i j}(0)=0, \quad \mathrm{~A}_{i}(0)=0, \quad \mathrm{~B}_{i j}(0)=\mathrm{B}_{i i}(0)=0, \quad \mathrm{~B}_{i j}(0)=0, \quad i, j=\overline{1, n}$. Conditional probabilities $\varphi_{i}(t), \psi_{i j}(t), \alpha_{i}(t)$ and $\beta_{i j}(t)$, according to (1)-(3), found from the relations

$$
\begin{gathered}
\varphi_{i}(t)=\left(1-f^{(i)}(t)\right)\left(p_{i 0}+\sum_{j=1}^{n} p_{i j} \varphi_{j}(t)\right), \psi_{i j}(t)=f^{(i)}(t) \delta_{i j}+\left(1-f^{(i)}(t)\right) \sum_{l=1}^{n} p_{i l} \psi_{l j}(t), \\
\alpha_{i}(t)=p_{i 0}+\sum_{j=1}^{n} p_{i j} \varphi_{j}(t),{ }^{\beta_{i j}(t)=\sum_{l=1}^{n} p_{i l} \psi_{l j}(t)}, i, j=\overline{1, n} .
\end{gathered}
$$

Solving the systems of linear equations (16) in the package Mathematica, analytical solutions can be obtained, but they are cumbersome already at $n=3$. For example, expression for the conditional probability of a time-dependent $\varphi_{1}(t)$, at $n=3$ has the form: 


$$
\begin{aligned}
& \varphi_{1}(t)=\left(\left(e^{-t} p_{13}\left(e^{-2 t} p_{22}-1\right)-e^{-3 t} p_{12} p_{23}\right)\left(e^{-4 t} p_{13} p_{30}-e^{-t} p_{10}\left(e^{-3 t} p_{33}-1\right)\right)-\right. \\
& \left.\quad-\left(e^{-3 t} p_{13} p_{20}-e^{-3 t} p_{10} p_{23}\right)\left(e^{-4 t} p_{13} p_{32}-e^{-t} p_{12}\left(e^{-3 t} p_{33}-1\right)\right)\right) / \\
& /\left(\left(e^{-t} p_{13}\left(e^{-2 t} p_{22}-1\right)-e^{-3 t} p_{12} p_{23}\right)\left(e^{-4 t} p_{13} p_{31}-\left(e^{-t} p_{11}-1\right) e^{-t}\left(e^{-3 t} p_{33}-1\right)\right)-\right. \\
& \left.\quad-\left(e^{-3 t} p_{13} p_{21}-e^{-2 t}\left(e^{-t} p_{11}-1\right) p_{23}\right)\left(e^{-4 t} p_{13} p_{32}-e^{-t} p_{12}\left(e^{-3 t} p_{33}-1\right)\right)\right),
\end{aligned}
$$

and for the probability $\psi_{21}(t)$ :

$$
\begin{gathered}
\psi_{21}(t)=\left(e^{4 t} p_{20}-e^{3 t} p_{11} p_{20}-e^{3 t} p_{21}+e^{4 t} p_{21}+e^{3 t} p_{10} p_{21}+p_{13} p_{21} p_{30}+e^{t} p_{23} p_{30}-\right. \\
-p_{11} p_{23} p_{30}-p_{13} p_{20} p_{31}-p_{23} p_{31}+e^{t} p_{23} p_{31}+p_{10} p_{23} p_{31}-e^{t} p_{20} p_{33}+p_{21} p_{20} p_{33}+ \\
\left.+p_{21} p_{33}-e^{t} p_{21} p_{33}-p_{10} p_{21} p_{33}\right) /\left(-e^{6 t}+e^{5 t} p_{11}+\right. \\
+e^{3 t} p_{12} p_{21}+e^{4 t} p_{22}-e^{3 t} p_{11} p_{22}+e^{2 t} p_{13} p_{31}-p_{13} p_{22} p_{31}+p_{12} p_{23} p_{31}+p_{13} p_{21} p_{32}+ \\
\left.+e^{t} p_{23} p_{32}-p_{11} p_{23} p_{32}+e^{3 t} p_{33}-e^{2 t} p_{11} p_{33}-p_{12} p_{21} p_{33}-e^{t} p_{22} p_{33}-p_{11} p_{22} p_{33}\right)
\end{gathered}
$$

Expression (13) takes the form

$$
\begin{aligned}
& \Psi_{n}(z, t)=a_{0}(t) \sum_{l_{1}=0}^{\infty} \ldots \sum_{l_{n}=0}^{\infty} \sum_{q_{1}=0}^{\infty} \ldots \sum_{q_{n}=0 r_{1}=0}^{\infty} \ldots \sum_{r_{n}=0}^{\infty} \prod_{i=1}^{n}\left[\frac{p_{0 i}^{l_{i}}}{l_{i} ! q_{i} ! r_{i} !} \times\right. \\
& \times\left(\prod_{j=1}^{n}\left(\frac{\lambda}{2}\left(\psi_{i j}(t) t^{2}-\int \psi_{i j}^{\prime}(t) t^{2} d t\right)\right)\right)^{l_{i}} \times \\
& \times\left(\mu_{i}\left(\alpha_{i}(t)\left(\frac{\sin \left(\omega_{i} t\right)}{\omega_{i}}+t\right)-\int \alpha_{i}^{\prime}(t)\left(\frac{\sin \left(\omega_{i} t\right)}{\omega_{i}}+t\right) d t\right)\right)^{q_{i}} \times \\
& \left.\times\left(\mu_{i} \prod_{j=1}^{n}\left(\beta_{i j}(t)\left(\frac{\sin \left(\omega_{i} t\right)}{\omega_{i}}+t\right)-\int \beta_{i j}^{\prime}(t)\left(\frac{\sin \left(\omega_{i} t\right)}{\omega_{i}}+t\right) d t\right)\right)^{r_{i}} z_{i}^{x_{i}+l_{i}-q_{i}-r_{i}+R}\right],
\end{aligned}
$$

after simplification we obtain:

$$
\begin{aligned}
\Psi_{n}(z, t)= & a_{0}(t) \sum_{l_{1}=0}^{\infty} \ldots \sum_{l_{n}=0}^{\infty} \sum_{q_{1}=0}^{\infty} \ldots \sum_{q_{n}=0_{r_{1}}=0}^{\infty} \ldots \sum_{r_{n}=0}^{\infty} \prod_{i=1}^{n}\left[\left(\frac{\lambda}{2} t^{2}\right)^{l_{i}} \frac{\mu_{i}^{q_{i}+r_{i}} p_{0 i}^{l_{i}}}{l_{i} ! q_{i} ! r_{i} !} \times\right. \\
\times & \left(\prod_{j=1}^{n} \int \psi_{i j}(t) t d t\right)^{l_{i}}\left(\int \alpha_{i}(t)\left(\cos \left(\omega_{i} t\right)+1\right) d t\right)^{q_{i}} \times \\
& \left.\left(\prod_{j=1}^{n} \int \beta_{i j}(t)\left(\cos \left(\omega_{i} t\right)+1\right) d t\right)^{r_{i}} z_{i}^{x_{i}+l_{i}-q_{i}-r_{i}+R}\right]
\end{aligned}
$$

Suppose we have found, for example, the probability of state It is the coefficient of $z_{1} z_{2} \cdot \ldots \cdot z_{n}$ in the expansion of $\Psi_{n}(z, t)$ in multiple series (17), so that when the degree of $z_{i}$ must satisfy the relation $x_{i}+l_{i}-q_{i}-r_{i}+R=1, i=\overline{1, n}$, it follows that 


$$
\begin{gathered}
q_{i}=x_{i}+l_{i}+\sum_{\substack{j=1 \\
j \neq i}} r_{j}-1, i=\overline{1, n}, q_{i}+r_{i}=x_{i}+l_{i}+\sum_{j=1} r_{j}-1, i=\overline{1, n}, \\
l_{i}+q_{i}+r_{i}=x_{i}+2 l_{i}+\sum_{j=1} r_{j}-1, i=\overline{1, n}, \sum_{i=1}^{n}\left(l_{i}+q_{i}+r\right)=\sum_{i=1}^{n}\left(x_{i}+2 l_{i}\right)+n(R-1) .
\end{gathered}
$$

Therefore, from (17) follows that

$$
\begin{aligned}
& P(1,1, \ldots, 1, t)=a_{0}(t) \sum_{l_{1}=0}^{\infty} \ldots \sum_{l_{n}=0 r_{1}=0}^{\infty} \sum_{r_{n}=0}^{\infty} \ldots \sum_{i=1}^{\infty}\left[\frac{p_{0 i}^{l_{i}}}{l_{i} !\left(x_{i}+l_{i}+\sum_{\substack{j=1 \\
j \neq i}} r_{j}-1\right) ! r_{i} !}\left(\frac{\lambda}{2} t^{2}\right)^{\sum_{i=1}^{n} l_{i}} \mu_{i}^{x_{i}+l_{i}+R-1} \times\right. \\
& \left.\times\left(\prod_{j=1}^{n} \int \psi_{i j}(t) t d t\right)^{l_{i}}\left(\int \alpha_{i}(t)\left(\cos \left(\omega_{i} t\right)+1\right) d t\right)^{x_{i}+l_{i}+\sum_{\substack{j=1 \\
j \neq i}} r_{j}-1}\left(\prod_{j=1}^{n} \int \beta_{i j}(t)\left(\cos \left(\omega_{i} t\right)+1\right) d t\right)^{r_{i}}\right] .
\end{aligned}
$$

We assume that $n=10$ and the initial time the network is in a state $(2,2,2,2,2,2,2,2,2,2,0)$. We find the probability of the state $P(1,1,1,1,1,1,1,1,1,1, t)$, using the formula (17). Let the intensities $\lambda(t)=\lambda t, \mu_{i}(t)=\mu_{i}\left[\cos \left(\omega_{i} t\right)+1\right]$, transition probabilities of messages are equal: $p_{0 i}=0.1, i=\overline{1,10}, \quad p_{10 i}=0.1, i=\overline{0,9}$, $p_{i 0}=0.5, i=\overline{1,9}, p_{i 10}=0.5, i=\overline{1,9}, p_{i i}=0, i=\overline{0,10}$. In addition, the intensity of the service messages in the systems are: $\mu_{i}=20.8, i=\overline{1,4}, \mu_{i}=10.22, i=\overline{4,9}$, $\mu_{10}=20.5$. The expression for the time-dependent probability of the state in the systems of the network obtained by on a computer using a mathematical calculation package Mathematica. Figure 1 shows a chart of this probability depending on the time $t$.

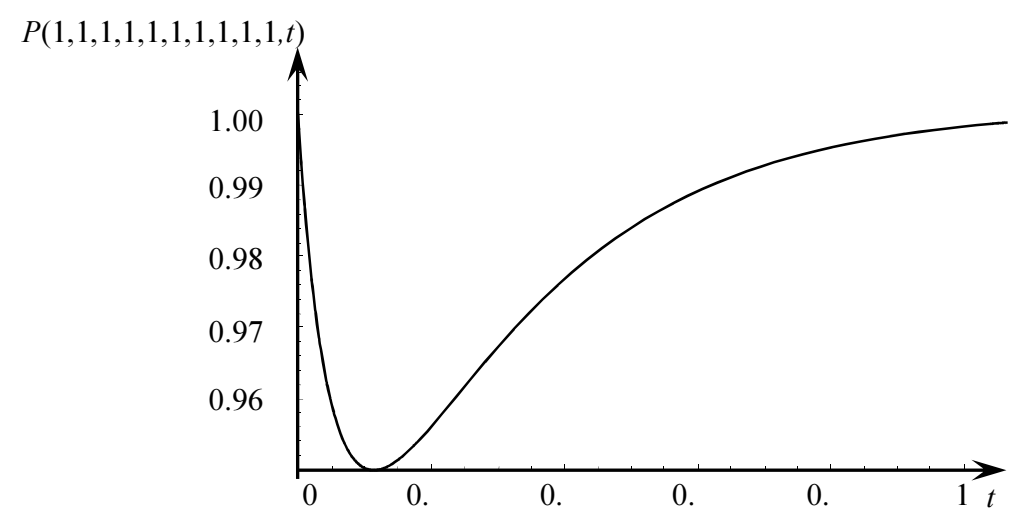

Fig. 1. The chart of probability of the state $P(1,1,1,1,1,1,1,1,1,1, t)$ 


\section{Example 2}

We will consider the network described in Example 1. Let $\lambda(t)=\lambda t$, $\mu_{i}(t)=\mu_{i}\left[\cos \left(\omega_{i} t\right)+1\right]$, transition probabilities of messages are equal: $p_{0 i}=0.1, i=\overline{1,10}, \quad p_{10 i}=0.1, i=\overline{0,9}, \quad p_{i 0}=0.5, i=\overline{1,9}, \quad p_{i 10}=0.5, i=\overline{1,9}$, $p_{i i}=0, i=\overline{0,10}$, intensity of service messages in the systems are: $\mu_{i}=20.8, i=\overline{1,4}$, $\mu_{i}=10.22, i=\overline{4,9}, \mu_{10}=20.5$. The average number of messages in the systems network (in the queue and service) at the initial time $t=0$ equally $N_{i}(0)=0$, $i=\overline{1,10}$. Equation (15) to find the average number of messages in the systems network when the network parameters and the conditional probabilities depend on the time takes the form:

$$
\begin{gathered}
N_{c}(t)=\exp \left\{-\sum_{i=1}^{n}\left[\frac{\lambda}{2}\left(t^{2}-\int \varphi_{i}(t) t d t\right) p_{0 i}+\right.\right. \\
\left.\left.+\mu_{i}\left(\frac{\sin \left(\omega_{i} t\right)}{\omega_{i}}+t-\int \beta_{i i}(t)\left(\cos \left(\omega_{i} t\right)+1\right) d t\right)\right]\right\} \times \sum_{k_{1}=1}^{\infty} \ldots \sum_{k_{n}=1}^{\infty} k_{c} \sum_{r_{1}=0}^{\infty} \ldots \sum_{r_{n}=0}^{\infty} \sum_{q_{1}=0}^{x_{1}-r_{1}+R-1} \cdots \sum_{q_{n}=0}^{x_{n}-r_{n}+R-1} \times \\
\times \prod_{i=1}^{n}\left[\frac{\lambda^{k_{i}-x_{i}+q_{i}+r_{i}-R} p_{0 i}^{l_{i}}}{\left(k_{i}-x_{i}+q_{i}+r_{i}-R\right) ! q_{i} ! r_{i} !}\left(\prod_{j=1}^{n} \int \psi_{i j}(t) t d t\right)^{k_{i}-x_{i}+q_{i}+r_{i}-R_{i}} \times\right. \\
\left.\times\left(\int \alpha_{i}(t)\left(\cos \left(\omega_{i} t\right)+1\right) d t\right)^{q_{i}}\left(\prod_{j=1}^{n} \int \beta_{i j}(t)\left(\cos \left(\omega_{i} t\right)+1\right) d t\right)^{r_{i}}\right]
\end{gathered}
$$

This example is designed on a computer using a mathematical calculation package Mathematica. Here are some of the values of the average number of applications in the systems of the network (in the queue and service) at time $t=0.2$, found using the program: $N_{1}(t)=0.547, \quad N_{2}(t)=0.323, \quad N_{3}(t)=0.429$, $N_{4}(t)=0.522, N_{7}(t)=0.742, N_{10}(t)=0.654$. Figure 2 shows a chart of the average number of messages in the QS $S_{3}$ depending on time $t$.



Fig. 2. The chart of changes of the average number of messages $N_{3}(t)$ in QS $S_{3}$ 


\section{References}

[1] Ivnitsky V., Theory of Queuing Network. Monograph, Fizmatlit, Moscow 2004 (in Russian).

[2] Malinkovsky Y., Queuing Networks with Bypass of Nodes. Automation and Remote Control 1991, 2, 102-110 (in Russian).

[3] Matalytski M., Queuing theory and its applications, Monograph., Grodno 2011.

[4] Matalytski M., Naumenko V., Analysis of queueing network with messages bypass of systems in transient behavior, Scientific Research of the Institute of Mathematics and Computer Science 2012, 11(2).

[5] Koluzaeva E., Finding the probabilities of states of models of information and computer networks with time-dependent parameters of flow and maintenance of operating under high load Vestnik GrSU. Ser. 2.2008, 3, 22-29 (in Russian).

[6] Statkevich S., Investigation of queuing networks with unreliable systems in the transient behavior, Vestnik of Tomsk State University. Series: Management, Computer Science and Informatics. 2012, 1, 112-125 (in Russian). 